\title{
Unfolding anti-tumor immunity: ER stress responses sculpt tolerogenic myeloid cells in cancer
}

\author{
Juan R. Cubillos-Ruiz ${ }^{1 *}$, Eslam Mohamed ${ }^{2}$ and Paulo C. Rodriguez ${ }^{3^{*}}$
}

\begin{abstract}
Established tumors build a stressful and hostile microenvironment that blocks the development of protective innate and adaptive immune responses. Different subsets of immunoregulatory myeloid populations, including dendritic cells, myeloid-derived suppressor cells (MDSCs) and macrophages, accumulate in the stressed tumor milieu and represent a major impediment to the success of various forms of cancer immunotherapy. Specific conditions and factors within tumor masses, including hypoxia, nutrient starvation, low pH, and increased levels of free radicals, provoke a state of "endoplasmic reticulum (ER) stress" in both malignant cells and infiltrating myeloid cells. In order to cope with ER stress, cancer cells and tumor-associated myeloid cells activate an integrated signaling pathway known as the Unfolded Protein Response (UPR), which promotes cell survival and adaptation under adverse environmental conditions. However, the UPR can also induce cell death under unresolved levels of ER stress. Three branches of the UPR have been described, including the activation of the inositol-requiring enzyme 1 (IRE1), the pancreatic ER kinase (PKR)-like ER kinase (PERK), and the activating transcription factor 6 (ATF6). In this minireview, we briefly discuss the role of ER stress and specific UPR mediators in tumor development, growth and metastasis. In addition, we describe how sustained ER stress responses operate as key mediators of chronic inflammation and immune suppression within tumors. Finally, we discuss multiple pharmacological approaches that overcome the immunosuppressive effect of the UPR in tumors, and that could potentially enhance the efficacy of cancer immunotherapies by reprogramming the function of tumor-infiltrating myeloid cells.
\end{abstract}

Keywords: ER stress, Unfolded Protein Responses, IRE1, XBP1, PERK, CHOP, Myeloid cells, Immunotherapy, Tumor immunology

\section{Background}

The Endoplasmic Reticulum (ER) plays a fundamental role in the homeostatic synthesis, folding and glycosylation of nascent transmembrane and secretory proteins [1]. In addition, the ER acts as the primary organelle for calcium storage and biosynthesis of lipids and sterols in eukaryotic cells [2]. The physiological activity of the ER is tightly controlled by intrinsic processes such as cell differentiation, proliferation status and activation signals, as well as by exogenous factors in the microenvironment

\footnotetext{
* Correspondence: jur2016@med.cornell.edu; Paurodriguez@Augusta.edu 'Weill Cornell Medicine, Department of Obstetrics \& Gynecology, Sandra and Edward Meyer Cancer Center, 1300 York Ave, E-907, New York, NY 10065, USA ${ }^{3}$ Department of Medicine, Georgia Cancer Center, Augusta University, 1410 Laney Walker Blvd, Room CN-4114, Augusta, GA 30912, USA

Full list of author information is available at the end of the article
}

[3]. For instance, hostile conditions in the tumor milieu such as hypoxia, nutrient starvation, low $\mathrm{pH}$, and free radicals can rapidly disrupt the protein folding capacity of the ER, thereby triggering a state of cellular "ER stress" [4]. The accumulation of misfolded proteins in the ER activates the Unfolded Protein Response (UPR), which is an integrated signaling pathway that attempts to restore the homeostasis of this organelle. The UPR drives multiple adaptive and survival processes, including the attenuation of de novo protein synthesis, the regulation of the ER membrane, the degradation of misfolded proteins, and the selective induction of mediators and chaperones that promote the correct folding of proteins [5]. However, when ER stress is severe and prolonged, the same UPR mediators that regulate survival can trigger the induction of cellular death [6]. Overactivation of UPR mediators has been 
implicated in several pathological processes, including cancer, diabetes, and cardiovascular and neurodegenerative diseases [4]. In addition, recent studies have demonstrated the importance of the UPR in the overall modulation of chronic inflammation in cancer [7-10]. In this review, we discuss how ER stress and aberrant activation of the UPR alter the function of malignant cells and cancer-associated myeloid cells, and how this process controls anti-tumor immunity. We also discuss various pharmacological approaches to overcome the immunosuppressive effect of ER stress in tumors and the potential of these strategies as new cancer immunotherapies.

\section{Review}

\section{ER stress sensors and the UPR}

The UPR plays a crucial role in mediating cellular adaptation to ER stress. Three major ER-localized transmembrane proteins trigger this adaptive pathway: the inositol-requiring enzyme 1 (IRE1), the pancreatic ER kinase (PKR)-like ER kinase (PERK), and the activating transcription factor 6 (ATF6) [4]. In the absence of ER stress, these three sensors are bound and maintained in an inactive form by the HSP70-type chaperone BiP/ GRP78 [11-13]. Because BiP exhibits a higher affinity for misfolded proteins, the induction of ER stress causes the dissociation of BiP from the sensors, leading to their activation and subsequent initiation of the UPR. The mechanisms by which the major mediators of the UPR regulate cellular responses under ER stress are as follow:

\section{IRE1}

The Type I ER transmembrane protein IRE1 is a dual enzyme with serine/threonine-protein kinase and endoribonuclease activity that exists in two conserved isoforms: IRE1 $\alpha$ and IRE1 $\beta$ [14, 15]. IRE1 $\alpha$ is ubiquitously expressed, whereas IRE1 $\beta$ expression is limited to the gut $[14,16]$. At steady state, the chaperone BiP maintains IRE1 $\alpha$ in its monomeric form, thereby impeding its activation. During ER stress, the accumulation of misfolded proteins titrate BiP away from IRE1 $\alpha$, allowing IRE1 $\alpha$ dimerization, autophosphorylation, and a conformational shift that licenses its $\mathrm{C}$-terminal endoribonuclease domain to excise 26 nucleotides from the X-box binding protein 1 (Xbp1) mRNA in the cytosol [17-19]. The spliced transcript is subsequently re-ligated by the tRNA ligase RtcB [20], resulting in a critical reading frame shift that allows the generation of the functionally mature XBP1. This transcription factor effectively alleviates ER stress by inducing the expression of chaperones, redox-dependent foldases, and glycosyltransferases. Beyond its canonical functions in the UPR, XBP1 can also modulate ER stress-independent, context-specific processes such as response to hypoxia [21], lipid metabolism [22], estrogen receptor activity [23] and the transcriptional induction of pro-inflammatory cytokines [24], among many others.

Although most of the IRE1 $\alpha$ signaling events are associated with the induction of pro-survival pathways, IRE1 $\alpha$ can also trigger apoptosis under severe or lethal ER stress. As such, IRE1 $\alpha$ can degrade non-Xbp1 mRNA targets through regulated IRE1 $\alpha$-dependent decay (RIDD), a phenomenon that has been previously associated with the induction of apoptosis [25]. Moreover, active IRE1 $\alpha$ complexes with the adaptor protein TNF-receptor-associated factor 2 (TRAF2), which recruits the apoptosis-signalregulating kinase (ASK1), leading to cell death or autophagy [26-28]. Additionally, IRE1 $\alpha$-linked apoptosis has been reported to be mediated through the activation of the c-Jun N-terminal kinase (JNK) and a subsequent inhibition of BCL2 family members [29]. Furthermore, activation of XBP1 through IRE1 $\alpha$ induces the expression of the HSP40 family member P58IPK, which binds and inhibits PERK, overcoming the PERK-mediated translational block [30]. Although this event can represent the termination of the UPR under transient ER stress, it may also trigger apoptosis under severe conditions of stress through the translation of pro-apoptotic mediators [31, 32]. Thus, IRE1 $\alpha$ can play a dual role in the cellular responses against ER stress by promoting both survival and cell death.

\section{PERK}

Under homeostatic conditions, the type I ER transmembrane protein PERK (or eIF2aK3) is maintained in an inactive form also through complexing with $\mathrm{BiP}$ [33]. After the induction of ER stress and release of BiP, PERK activates through oligomerization and autophosphorylation, leading to the phosphorylation of various PERK substrates, including the eukaryotic translation initiation factor 2 alpha (eIF2 $\alpha$ ), the NF-E2-related factor 2 (Nrf2), the forkhead box O proteins (FOXO), and the second messenger diacyglycerol (DAG) [34]. The increased susceptibility of PERK null primary cells and tumor cells to ER stress-induced cell death suggests the major role of PERK in pro-survival mechanisms [35, 36]. The best-characterized PERK-linked effect is the phosphorylation of eIF2 $\alpha$, which serves as a common regulator of the integrated stress responses in cells. In addition to PERK, three different kinases, the double-stranded RNAdependent protein kinase (PKR), the hemin-regulated inhibitor (HRI), and the nutrient starvation activated kinase GCN2, phosphorylate eIF2 $\alpha$ in response to specific forms of stress [37]. Phospho-eIF2 $\alpha$ inhibits nucleotide exchange on the eIF2 complex, attenuating translation of most mRNAs, thereby alleviating additional sources of ER stress [37]. In addition, it increases the Cap-independent expression of a limited number of proteins that eventually control the cell fate during stress, including the activating transcription factor 4 (ATF4). Thus, phosphorylation of 
eIF2 $\alpha$ by PERK serves as a major mechanism to decrease protein synthesis and thereby counter the accumulation of misfolded proteins in the stressed ER. In addition, active PERK phosphorylates Nrf2, which then translocates to the nucleus and induces the expression of multiple cellular redox transcripts that alleviate the effects of stress-induced reactive oxygen species (ROS) [38]. Also, activation of FOXO proteins by PERK negatively regulates AKT activity and therefore converts stressed cells from anabolic metabolic programs into those leading to nutrient catabolism [39]. Thus, the activation of PERK plays a fundamental role in the metabolic adaptation of cells to ER stress.

Phosphorylation of eIF2 $\alpha$ induces the activation of ATF4 that directly regulates the survival of the stressed cells through the induction of autophagy. Interestingly, ATF4 induction after uncontrolled or chronic ER stress regulates the expression of the pro-apoptotic protein CAAT/enhancer binding protein (C/EBP) homologous protein (CHOP/Ddit3), which plays a key role in the induction of cell death by stress [40]. The mechanism by which PERK activity plays a dual role in the survival of stressed cells has been recently demonstrated. The induction of ATF4 after PERK activation results in the transient expression of the microRNA miR-211, which temporarily blocks the transcription of pro-apoptotic CHOP. However, after the expiration of miR-211, CHOP transcription proceeds and the cells undergo apoptosis [41]. Therefore, similar to the role played by IRE1 $\alpha$, the activation of PERK can mediate pro-survival or pro-apoptotic effects.

\section{ATF6}

ATF6 is an ER-resident type II transmembrane protein that exists as 2 homologs (ATF6 $\alpha$ and ATF6 $\beta$ ) and serves as a precursor for a cytoplasmic N-terminal bZIP transcription factor [42]. Upon dissociation from BiP, ATF6 $\alpha$ translocates to the Golgi apparatus via coat protein COPII-covered vesicles where it results cleaved by site 1 and site 2 proteases, enabling its transcription factor potential [13]. ATF6 $\alpha$ target genes regulate the folding and glycosylation of de novo proteins, thereby regulating the survival of stressed cells [43]. In addition, several common targets of ATF $6 \alpha$ are also regulated by XBP1, suggesting potential overlapping effects of IRE $1 \alpha$ and ATF6 $\alpha$. Although the role of ATF6 $\alpha$ and ATF6 $\beta$ upon ER stress remains less critical than that induced by IRE1 $\alpha$ and PERK, the knockdown of Atf6 results in lower survival rates after specific chemically-induced ER stress, indicating that ATF6 $\alpha$ is indeed protective in the responses induced by pharmacological ER stress [43].

\section{Role of the UPR in malignant cells}

The key interaction between the UPR and tumorigenesis has been comprehensively discussed in previous reviews
[1, 4, 5, 34]. Malignant cells thrive under ER stressinducing conditions such as hypoxia, nutrient deprivation, and low $\mathrm{pH}$. In addition, cancer cells generate reactive metabolic byproducts that avidly modify ER-resident proteins and chaperones. Notably, the induction of various UPR-related factors has been commonly reported in patients with various cancer types and their overexpression usually correlates with poor prognosis and resistance to therapy [21, 44-46]. Interestingly, treatment of tumorbearing mice with the ER stress inducer thapsigargin increased tumor growth, whereas global UPR inhibition using chemical chaperones, such as 4-Phenylbutyric acid (4-PBA) or tauroursodeoxycholic acid (TUDCA), delayed tumor progression and metastasis [9, 47].

Seminal studies have determined the cancer cellintrinsic protumoral role of the IRE1 $\alpha-\mathrm{XBP} 1$ and the PERK-eIF $2 \alpha$ pathways in vivo. Implantation of malignant cells or transformed fibroblasts lacking IRE1 $\alpha /$ XBP1 or PERK/eIF2 $\alpha$ in mice resulted in reduced tumor growth, which was attributed to low angiogenesis and increased sensitivity of the cancer cells to ER stress inducers, including hypoxia and high levels of ROS [35]. Accordingly, targeting IRE1 $\alpha$ or PERK signaling in vivo with specific small-molecule inhibitors has shown significant therapeutic effects in various preclinical models of disease [48-52]. More recently, XBP1 was demonstrated to foster triple negative breast cancer progression by cooperating with HIF $1 \alpha$ to support tumor-initiating cell function and metastatic capacity under hypoxia [21]. XBP1 contributes to the pathogenesis of multiple myeloma [53], and has been implicated in cancer cell de-differentiation, susceptibility to oncovirus infection and the epithelial-to-mesenchymal transition [54]. Andrew $\mathrm{Hu}$ and colleagues have elegantly demonstrated constitutive IRE1 $\alpha$-XBP1 activation in chronic lymphocytic leukemia cells, which promoted their pathogenesis in vivo [48]. In addition, inhibiting IRE1 $\alpha$ function by overexpressing a dominant negative IRE1 $\alpha$ variant significantly increased overall host survival by decreasing tumor growth rate and angiogenesis in a model of glioma [55]. Recent studies have also indicated that IRE1 $\alpha$-XBP1 signaling supports the aggressiveness of pancreatic cancer cells in xenograft models [56].

Similar to the effect induced by IRE1 $\alpha$-XBP1 signaling, the activation of PERK-eIF2 $\alpha$ has also been implicated in the development of several malignancies, including breast, lung, and liver carcinoma [36, 47]. In those models, deletion of Perk rendered malignant cells highly susceptible to the cell death induced after exposure to hypoxia, DNA damage, low levels of nutrients, and high levels of reactive oxygen species [57]. Furthermore, the absence of PERKeIF2 $\alpha$ signaling impaired the ability of breast cancer cells to migrate and invade, thereby decreasing their ability to metastasize in vivo $[49,58,59]$. Therefore, the inhibition 
of PERK resulted in cancer cell apoptosis and significant anti-tumor effects [43]. As such, silencing of Perk increased the therapeutic efficacy of treatments based on the depletion of amino acids in T cell leukemia [60], and sensitized chronic myeloid leukemia (CML) cells to the apoptosis induced by the BCR/ABL inhibitor, imatinib mesylate [61]. Thus, the intrinsic effects of a controlled UPR in cancer cells appear to favor tumor growth and metastasis through the promotion of malignant cell survival, angiogenesis and chemoresistance, thus justifying the use of specific UPR inhibitors for the treatment cancer.

Although activation of the UPR has been primarily associated with cancer cell survival and tumor progression, some studies suggest that molecular factors in this pathway could also suppress tumor development in certain contexts. For instance, increased oncogenic transformation has been evidenced in fibroblasts after inhibiting the PERK target eIF2 $\alpha$ [62], and increased proliferation and mammary tumor formation has been reported upon expression of a dominant-negative form of PERK in mammary epithelial cells [63]. Furthermore, in the context of acute myeloid leukemia, increased expression of ER stress response markers correlates with better prognosis in patients with this disease [64]. Taken together, these studies indicate that the effects of the UPR in cancer cells is context-dependent and that variables such as the stage of cancer progression and the cellular source of malignancy are critical determinants of whether this pathway plays either a pro-tumorigenic or anti-tumoral role.

\section{ER-stressed cancer cells efficiently manipulate myeloid functions}

Although the effect of the UPR in the survival/death of malignant cells has been extensively studied during the last decade, its role in the modulation of anti-tumor immunity has remained minimally characterized. Superior tolerogenic activity is observed in tumor-infiltrating myeloid cells compared with those located outside the tumors, suggesting a role for the tumor-stressed microenvironment in the control of myeloid cell function $[65,66]$. Initial in vitro studies reported paracrine effects of tumor cells undergoing ER stress on dendritic cells (DCs), macrophages, and myeloid-derived suppressor cells (MDSCs). Pharmacological induction of UPR in cancer cells triggered "transmissible" ER stress in myeloid cells, as evidenced by the upregulation of UPR-related elements in these innate immune cells upon exposure to supernatants from treated cancer cells [67]. In this system, induction of ER stress markers in myeloid cells correlated with their decreased ability to induce $\mathrm{T}$ cell responses, elevated expression of suppressive factors such as arginase I and prostaglandin E2 $\left(\mathrm{PGE}_{2}\right)$, and upregulation of various cytokines including IL-6, IL-8, TNFo, and IL-23 [67] [10]. The impairment of myeloid cells exposed to supernatants from ER-stressed cancer cells to activate $\mathrm{T}$ cell responses was associated with a reduction in their antigen-presenting capacity [68]. Moreover, DCs conditioned in vitro with supernatants from ER-stressed cancer cells were transformed into MDSCs and facilitated tumor growth after adoptive transfer into tumor-bearing mice [10] (Fig. 1). While these studies suggested that ER-stressed cancer cells release soluble factors that more efficiently modulate immune cell function, it remained mechanistically and functionally elusive whether myeloid-intrinsic UPR factors were indeed responsible for the correlative changes described. Interestingly, administration of the ER stressor thapsigargin to tumor-bearing mice accelerated cancer progression and enhanced the accumulation and immunosuppressive capacity of MDSC, a process that could be attenuated upon in vivo treatment with the ER stress chemical chaperone, 4-PBA [9]. PERK has been implicated in blocking the effects of type 1 interferon potentially through direct regulation of the interferon receptor [69]. Previous results also showed that activation of PERK and the subsequent phosphorylation of eIF2 $\alpha$ increased the activity of NF-kB by controlling the translation, but not the degradation, of the NF-kB inhibitor IkB [70]. Similarly, activation of IRE1 $\alpha$ and ATF6 induced the phosphorylation of IkB and the subsequent activation of NF-kB in a manner dependent upon TRAF2 and Akt90, respectively [70, 71]. However, the potential interaction between the UPR and NF-kB in myeloid cells within tumors remains to be explored. These studies indicate that cancer cells undergoing ER stress can avidly modulate the phenotype of tumor-infiltrating myeloid cells.

\section{Cancer cell-intrinsic ER stress and immunogenic cell death (ICD)}

Chemotherapeutic agents of the anthracycline family have been shown to trigger the UPR in cancer cells and this process was associated with the induction of immunogenic cell death (ICD), activation of myeloid cell function, and protective anti-tumor immunity [72]. Nonetheless, it remains unclear how the induction ER stress in malignant cells could result in the development of suppressive or immunogenic responses. ICD induction by ER stress appears to be mediated through a significant elevation of ROS levels and a subsequent activation of the NLRP3inflammasome [4, 73]. However, the accumulation of ROS also remains as a major mechanism of $\mathrm{T}$ cell suppression by myeloid cells in tumors [74]. The fine balance between the levels of ROS and the specific ROS mediators could explain the opposite effects induced by stressed cancer cells on anti-tumor immunity (Fig. 2). Alternatively, the different consequences of tumor cells undergoing ER stress could also be explained by the 


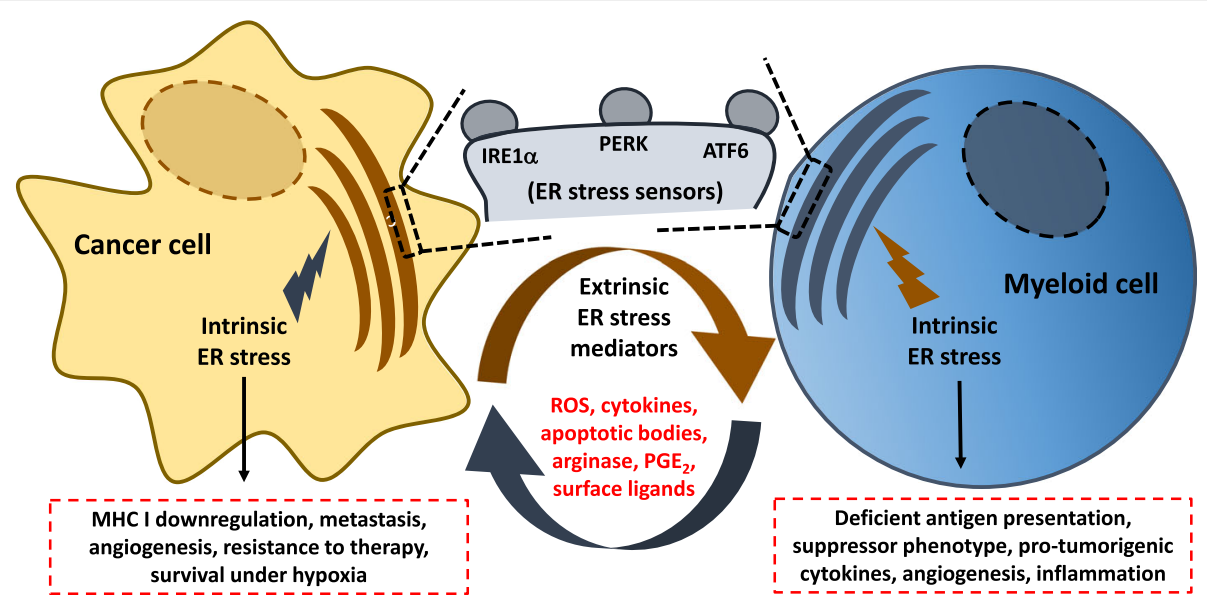

Fig. 1 Hostile conditions in the tumor microenvironment such as hypoxia, nutrient deprivation and ROS can provoke ER stress and trigger the UPR in various tumor-resident cell types. Intrinsic ER stress responses in cancer cells ensure their survival under hypoxic conditions, increase expression of pro-angiogenic factors, promote metastasis and inhibit the presentation of their own antigens. Myeloid-intrinsic ER stress responses mediate reprogramming towards immunosuppressive and tolerogenic phenotypes. Induction of ER stress in myeloid cells may occur via transmissible factors released by ER-stressed cancer cells in the same milieu. Intracellular generation and accumulation of lipid peroxidation byproducts can further elicit intrinsic ER stress responses in myeloid cells. ER stress sensors therefore emerge as attractive targets for developing new immunotherapeutic approaches that may synergize with standard cancer treatments

simultaneous development of suppressive and immunogenic UPR in different subsets within the malignant cell population. Another plausible explanation is that moderate but sustained ER stress triggers immunosuppressive effects, whereas a robust/lethal UPR could result in ICD (Fig. 2). Interestingly, superior anti-tumor immune responses were observed in mice injected with BiP-deficient fibrosarcoma cells, presumably due to lethal overactivation of ER stress sensors that promotes ICD [75]. Hence, sustained ER stress responses occurring in transformed cells could promote immunosuppression, while the dramatic overactivation of the UPR upon acute chemo- or radiotherapy regimens may promote immunostimulatory responses (Fig. 2). Strikingly, however, XBP1 was recently

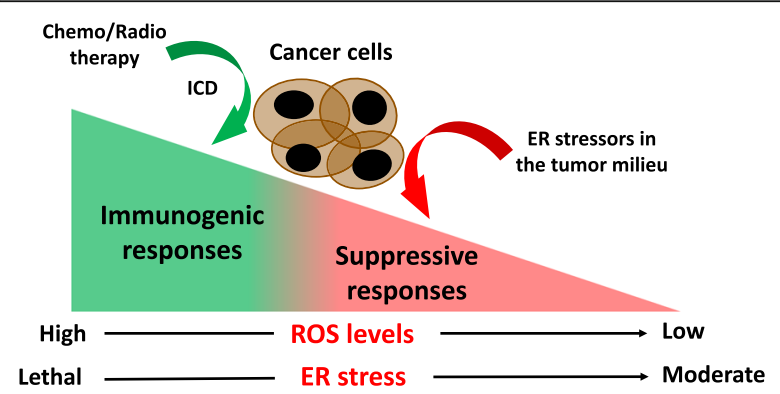

Fig. 2 The severity of ER stress and the levels of ROS in cancer cells can determine the outcome of immune responses within the tumor milieu. Intense ER stress responses induced by chemo- or radiotherapy increase ROS in cancer cells to levels that can promote immunogenic cell death (ICD), thus enhancing anti-tumor immunity. Moderate but sustained ER stress responses in cancer cells support tolerogenic and immunosuppressive functions in tumor-infiltrating myeloid cells, a process that cripples anti-cancer immunity shown to prevent ICD in metastatic colorectal cancer cells upon combination treatment with epidermal growth factor receptor blockers and chemotherapy [76].

\section{Intrinsic roles of the UPR in tumor-associated myeloid cells}

Elevated expression of UPR mediators in tumors correlated with stage, aggressiveness, and low survival in patients with different malignancies. However, the link between the induction of ER stress in the tumor stroma and immunosuppression in individuals with cancer has not been appreciated over the last 10 years. Initial studies showed the role of UPR in the regulation of inflammation through modulation of the production of ROS and the activation of NF-kB, Jnk and IRF3 [1]. Most recently, however, various groups have demonstrated that sustained ER stress responses also act as crucial drivers of myeloid cell dysfunction in tumors [7, 8, 77].

IRE1 $\alpha-\mathrm{XBP} 1$ signaling is required for the optimal homeostatic differentiation of plasma cells, eosinophils and some DC populations [78-80]. Optimal TLRdriven pro-inflammatory cytokine production in macrophages has been demonstrated to be mediated by XBP1 [24]. In a model of acute lung injury, neutrophils infiltrating early lesions exhibited signs of ER stress, and XBP1 expression by this specific myeloid population was required for disease progression [81]. Interestingly, the potential role of this arm of the UPR in controlling the regulatory phenotype of tumor-associated myeloid cells has recently emerged as a key mediator of immune suppression in cancer (Fig. 3). In ovarian cancer, dysfunctional tumor-associated DCs (tDCs) showed robust 


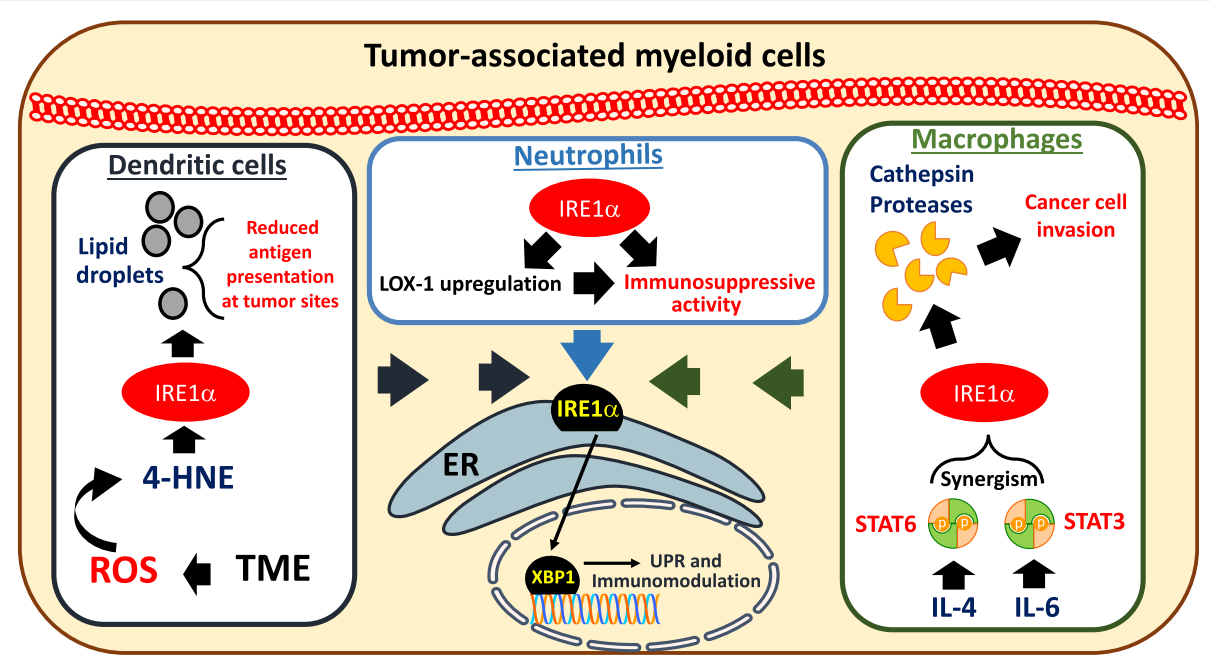

Fig. 3 IRE1a-XBP1 is one of the arms of UPR that polarizes tumor-infiltrating myeloid cells into highly immunosuppressive populations. Over activation of IRE1 a-XBP1 pathway by the byproduct adduct 4-hydroxy-trans-2-nonenal (4-HNE) in the tumor microenvironment (TME) shifts tumor-infiltrating dendritic cells towards a tolerogenic phenotype that promotes cancer cell growth. IRE1a-XBP1 activation upregulates lectin-type oxidized LDL receptor-1 (LOX-1) that converts high density anti-tumor neutrophils to low density immunosuppressive polymorphonuclear myeloid cells (PMNMDSCs). IL-4 and IL-6 signals synergize with IRE1a-XBP1 to enhance the ability of tumor-associated macrophages to secret Cathepsin proteases, which facilitate cancer cell invasion and metastasis

expression of ER stress markers and sustained activation of the IRE1 $\alpha$ - XBP1 arm of the UPR, compared with DCs residing in non-tumor locations [7]. Persistent ER stress responses in tDCs were provoked by intracellular ROS that promoted lipid peroxidation and subsequent generation of reactive aldehyde byproducts such as 4-hydroxynonenal (4-HNE), which modified several ER-resident chaperones and proteins [7]. Treatment of tDCs with ROS-scavenging vitamin $\mathrm{E}$ or hydrazine derivatives that avidly sequester 4-HNE ameliorated ER stress responses in tDC [7]. Conditional deletion of $X b p 1$ in DCs resulted in delayed ovarian cancer progression and this process was mediated through the induction of protective $\mathrm{T}$ cell anti-tumor immunity. Additional experiments further confirmed that tDCs lacking XBP1 were immunostimulatory rather than tolerogenic. Mechanistically, abnormal activation of XBP1 metabolically reprogrammed DCs towards aberrant triglyceride biosynthesis and uncontrolled lipid accumulation, a process that was associated with reduced tDC antigen-presenting capacity. Interestingly, aberrant lipid accumulation and the production of oxidized fatty acids are common tolerogenic characteristics of tumor-infiltrating DCs and MDSCs [82-84]. Consistent with the immunogenic effects induced upon deleting or silencing $X b p 1$ in tDCs, targeting lipid uptake or inhibiting key mediators of fatty acid oxidation has been shown to boost anti-cancer immunity by enhancing myeloid cell function in the tumor microenvironment [82-84]. While there is a clear interaction between the induction of ER stress and the metabolic reprogramming of myeloid cells in tumors, it remains unknown whether the tolerogenic effects induced by the accumulation of lipids in myeloid cells are solely mediated through IRE1 $\alpha-\mathrm{XBP} 1$ signaling or whether additional mediators participate in this process. Nevertheless, recent studies by Gabrilovich and colleagues have elegantly reinforced the crucial immunoregulatory role of aberrant IRE1 $\alpha$-XBP1 signaling in human cancer-associated myeloid cells [85]. In diverse human cancer specimens, upregulation of ER stress-related gene signatures and surface expression of the lectin-type oxidized LDL receptor-1 (LOX-1) distinguished highdensity neutrophils from low-density immunosuppressive polymorphonuclear MDSCs (PMN-MDSCs). Strikingly, pharmacological induction of ER stress in human neutrophils rapidly triggered LOX-1 upregulation and transformed them into immunosuppressive cells in an IRE1 $\alpha /$ XBP1-dependent manner. These recent studies indicate that the sustained activation of the IRE1 $\alpha$-XBP1 arm of the UPR promotes immunosuppression in cancer hosts by modulating the activity of tumor-associated DC, neutrophils, and MDSCs. Furthermore, a recent study showed that IRE1 $\alpha$-XBP1 signaling also shapes the pro-tumoral attributes of macrophages in cancer [86]. Through synergism between the IL-4 and IL- 6 signaling pathways to activate IRE1 $\alpha$, tumor-associated macrophages acquire a secretory phenotype that enables the infiltration of metastatic cancer cells via Cathepsin proteases.

In addition to the role of IRE1 $\alpha$-XBP1 in the suppressive function of tumor-infiltrating myeloid cells, recent studies have demonstrated a major function for the UPR downstream target $\mathrm{CHOP}$ as a key regulator of MDSC activity and turnover in tumors [77]. While the pro-apoptotic 
effect CHOP has been appreciated for years, it is now evident that it can also regulate other cellular functions independent of the induction of apoptosis. In fact, CHOP expression controlled the polarization of macrophages into "alternatively activated" cells and directly regulated the expression of various cytokines, including IL-23, IL$1 \beta$, and IL-6 [87-89]. Interestingly, CHOP levels can be increased not only upon activation of the UPR, but also through other immunoregulatory mechanisms, including nutrient starvation, TLR agonists, and increased ROS accumulation, suggesting its common involvement in multiple stress pathways. Elevated $\mathrm{CHOP}$ expression was found in MDSCs infiltrating mouse and human tumors, which directly correlated with the ability of MDSC to impair $\mathrm{T}$ cell responses $[8,77]$. Interestingly, the injection of CHOP-competent cancer cells into systemic Chop-deficient mice or Chop-null bone marrow chimeras resulted in a significant anti-tumor effect mediated by $\mathrm{CD}^{+} \mathrm{T}$ cells, suggesting the importance of hematopoietic-intrinsic $\mathrm{CHOP}$ in tumor growth and tumor-induced tolerance [77]. Furthermore, MDSCs isolated from tumor-bearing mice devoid of $\mathrm{CHOP}$ exhibited reduced ability to block $\mathrm{T}$ cell responses and impaired expression of major inhibitory pathways, while demonstrating an extraordinary ability to prime $\mathrm{T}$ cell proliferation and induce anti-tumor effects. Additional studies showed the potential role of $\mathrm{CHOP}$ in the regulation of $C / E B P \beta$, a pathway known to globally regulate MDSC function. This resulted in an increased production of IL- 6 that played a primary role in the effects induced by CHOP. Thus, the inhibition of CHOP could represent a major strategy to overcome the tolerogenic function of MDSCs and other myeloid suppressive cells in tumors. Similar to the effect of the endogenously produced ROS in the activation of IRE1 $\alpha$-XBP1 in tDCs [7], we found that pharmacological scavenging of ROS prevented the induction of $\mathrm{CHOP}$ in tumor-associated myeloid cells [77], suggesting the common role of ROS in the induction of UPR in cancer-infiltrating myeloid cells. Although the induction of CHOP after ER stress is primarily mediated through ATF4, it remains unknown the role of the CHOP-independent ATF4 effects in tumorassociated myeloid cells. Nevertheless, a seminal study by Condamine and colleagues showed the role of ER stress in the regulation of MDSC survival in tumors [8]. Induction of ER stress was detected preferentially in tumorinfiltrating MDSCs and promoted MDSC apoptosis through TNF-related apoptosis induced ligand receptor 2 (DR5) and caspase 8 activation [14]. Thus, DR5 agonists could be considered as potential strategies for controlling MDSC generation in cancer. Interestingly, deletion of Chop also regulated MDSC turnover, as a delayed MDSC apoptosis and extended MDSC survival rates were found in tumor-infiltrating MDSCs lacking this UPR mediator, compared with CHOP-sufficient controls [77]. Taken together, these recent findings suggest that ER stress responses driven by IRE1 $\alpha$-XBP1 and $\mathrm{CHOP}$ play a major role in the regulation of myeloid cell activity and survival in tumors. It remains elusive, however, whether the ATF6 arm of the UPR also contributes to myeloid cell dysfunction in cancer.

\section{Therapeutic approaches to overcome detrimental ER stress responses in tumor-associated myeloid cells}

Since the UPR appears to regulate anti-tumor immunity while promoting the intrinsic aggressiveness of malignant cells, it is conceivable that therapies aimed at attenuating ER stress or targeting UPR mediators may have a potent double-whammy effect against cancer. Chemical chaperones that prevent ER stress, such as TUDCA and 4-PBA, have shown promising therapeutic effects in preclinical cancer models. However, the consequence of treatment with these compounds on the global tumor immunoenvironment remains unknown. Additional efforts have been made to develop specific small-molecule inhibitors or nanoparticle-encapsulated siRNAs targeting UPR mediators. Compounds inhibiting the endoribonuclease domain of IRE1 $\alpha$, including STF083010, 3-ethoxy-5,6-dibromosalicylaldehyde, $4 \mu 8 \mathrm{C}$, MKC-3946, toyocamycin, and B-I09, can block Xbp1 splicing and activation in a dose dependent manner, especially in vitro [90]. Some of these compounds have been tested in vivo and demonstrated anti-tumor effects by directly affecting the cancer cell. While the immunotherapeutic capacity of these inhibitors has not been tested in vivo, delivery of nanoparticles encapsulating Xbp1-targeting siRNA into mice bearing metastatic ovarian carcinoma transformed tDCs into highly immunogenic cells capable of inducing protective $\mathrm{T}$ cell responses that extend host survival [7]. Treatment of tumor-bearing mice with the PERK small-molecule inhibitors GSK2656157 or GSK2606414 has also resulted in significant anti-tumor effects $[49,91]$, but it remains unknown whether these compounds could additionally relieve immunosuppression in the tumor microenvironment by controlling activation in myeloid cells, without inducing systemic toxicity. While these studies suggest the potential of targeting the UPR in cancer, disruptive medicinal chemistry approaches are urgently needed to generate more selective, potent and stable inhibitors of ER stress sensors for in vivo use.

A major impediment to the success of current immunotherapies is the accumulation of suppressive myeloid cells that prevent the generation and expansion of tumoricidal $\mathrm{T}$ cells [92]. Therapies based on targeting UPR mediators could be potentially used to reprogram suppressive myeloid populations into cells that activate anti-tumor immunity in situ. These approaches could be useful to relieve or diminish tumor-induced 
immunosuppression prior to treatment with other immunotherapies such as checkpoint blockade, adoptive $\mathrm{T}$ cell transfer or therapeutic vaccination. In summary, future studies on the role of the UPR in tumor-associated myeloid cells are expected to have a significant impact in the development of new immunotherapies that more effectively confront lethal cancers in the clinic.

\section{Conclusions}

Controlling the accumulation and detrimental activity of immunosuppressive myeloid cells in cancer patients emerges as a fundamental requirement for the success of cancer immunotherapies. However, interventions that effectively and permanently abolish the major regulatory effect or the accumulation of myeloid cells in tumors are lacking. Sustained ER stress responses have been demonstrated to promote malignant progression and metastasis. Further, recent studies revealed an additional role for endogenous ER stress and the UPR in regulating the function, expansion and differentiation of suppressive myeloid cells in cancer hosts. The activation of the UPR in myeloid cells can directly occur in response to the stressful tumor microenvironment or may be transmitted from neighboring ER-stressed cancer cells. Since disabling some ER stress sensors and UPR mediators can reprogram suppressive myeloid cells into cells that induce protective antitumor immunity, new interventions capable of controlling this pathway in vivo could improve the effectiveness of emerging cancer immunotherapies. We therefore propose that understanding the cellular and molecular effects of ER stress in tumor-associated myeloid cells will be crucial for developing more rational and hopefully definitive immunotherapies against lethal cancers.

\section{Abbreviations}

4-HNE: 4-hydroxynonenal; 4-PBA: 4-Phenylbutyric acid; ASK1: Apoptosissignal-regulating kinase; ATF4: Activating transcription factor 4; ATF6: Activating transcription factor 6; C/EBPB: CAAT/enhancer binding protein beta; CHOP: C/EBP homologous protein; CML: Chronic myeloid leukemia; DAG: Diacyglycerol; DCs: Dendritic Cells; DR5: TNF-related apoptosis induced ligand receptor 2; elF2a: Eukaryotic translation initiation factor 2 alpha; ER: Endoplasmic reticulum; FOXO: Forkhead box O protein; GCN2: Nutrient starvation activated kinase; HRI: Hemin-regulated inhibitor; ICD: Immunogenic cell death; IRE1: Inositol-requiring enzyme 1; JNK: c-Jun Nterminal kinase; LOX-1: Lectin-type oxidized LDL receptor-1; MDSCs: Myeloidderived suppressor cells; Nrf2: NF-E2-related factor 2; PERK: Pancreatic ER kinase (PKR)-like ER kinase; PGE 2 : Prostaglandin E2; PKR: Double-stranded RNA-dependent protein kinase; PMN-MDSCs: Polymorphonuclear MDSCs; RIDD: Regulated IRE1a-dependent decay; ROS: Reactive oxygen species; tDCs: Tumor-associated DCs; TRAF2: TNF-receptor-associated factor 2; TUDCA: Tauroursodeoxycholic acid; UPR: Unfolded Protein Response; XBP1: X-box binding protein 1

\section{Acknowledgements}

N/A

\section{Funding}

This work was partially supported by the Ovarian Cancer Academy-EarlyCareer Investigator Award of the Department of Defense and the Stand Up to Cancer IRG to J.R.C-R, and by National Institutes of Health (NIH) R01CA184185 to P.C.R.

\section{Availability of data and material}

N/A

Authors' contributions

J.R.C-R, E.M, and P.C.R contributed to the background research, design and preparation of the figures, and writing of this review. All authors have read and approved the final manuscript.

\section{Competing interests}

J.R.C-R is co-founder of and scientific advisor for Quentis Therapeutics, Inc.

\section{Consent for publication}

All authors state that this manuscript has not been published and is not under consideration for publication elsewhere.

\section{Ethics approval and consent to participate}

N/A

\section{Author details}

Teill Cornell Medicine, Department of Obstetrics \& Gynecology, Sandra and Edward Meyer Cancer Center, 1300 York Ave, E-907, New York, NY 10065, USA ${ }^{2}$ Georgia Cancer Center, Augusta University, 1410 Laney Walker Blvd, Room CN-4125A, Augusta, GA 30912, USA. ³ Department of Medicine, Georgia Cancer Center, Augusta University, 1410 Laney Walker Blvd, Room CN-4114, Augusta, GA 30912, USA.

Received: 1 September 2016 Accepted: 12 December 2016 Published online: 17 January 2017

\section{References}

1. Janssens S, Pulendran B, Lambrecht BN. Emerging functions of the unfolded protein response in immunity. Nat Immunol. 2014;15:910-9.

2. Walter $\mathrm{P}$, Ron $\mathrm{D}$. The unfolded protein response: from stress pathway to homeostatic regulation. Science. 2011;334:1081-6.

3. Ron D, Harding HP. Protein-folding homeostasis in the endoplasmic reticulum and nutritional regulation. Cold Spring Harb Perspect Biol. 2012;4:4-16.

4. Bettigole SE, Glimcher LH. Endoplasmic reticulum stress in immunity. Annu Rev Immunol. 2015;33:107-38.

5. Chevet E, Hetz C, Samali A. Endoplasmic reticulum stress-activated cell reprogramming in oncogenesis. Cancer Discov. 2015;5:586-97.

6. Tabas I, Ron D. Integrating the mechanisms of apoptosis induced by endoplasmic reticulum stress. Nat Cell Biol. 2011;13:184-90.

7. Cubillos-Ruiz JR, Silberman PC, Rutkowski MR, Chopra S, Perales-Puchalt A, Song M, et al. ER Stress Sensor XBP1 Controls Anti-tumor Immunity by Disrupting Dendritic Cell Homeostasis. Cell. 2015;161:1527-38.

8. Condamine T, Kumar V, Ramachandran IR, Youn Jl, Celis E, Finnberg N, et al. ER stress regulates myeloid-derived suppressor cell fate through TRAIL-Rmediated apoptosis. J Clin Invest. 2014:124:2626-39.

9. Lee BR, Chang SY, Hong EH, Kwon BE, Kim HM, Kim YJ, et al. Elevated endoplasmic reticulum stress reinforced immunosuppression in the tumor microenvironment via myeloid-derived suppressor cells. Oncotarget. 2014:5:12331-45.

10. Mahadevan NR, Anufreichik V, Rodvold JJ, Chiu KT, Sepulveda H, Zanetti M. Cell-extrinsic effects of tumor ER stress imprint myeloid dendritic cells and impair CD8(+) T cell priming. PLoS One. 2012;7:e51845.

11. Bertolotti A, Zhang Y, Hendershot LM, Harding HP, Ron D. Dynamic interaction of BiP and ER stress transducers in the unfolded-protein response. Nat Cell Biol. 2000;2:326-32.

12. Okamura K, Kimata $Y$, Higashio H, Tsuru A, Kohno K. Dissociation of Kar2p/ BiP from an ER sensory molecule, Ire1p, triggers the unfolded protein response in yeast. Biochem Biophys Res Commun. 2000;279:445-50.

13. Ye J, Rawson RB, Komuro R, Chen X, Dave UP, Prywes R, et al. ER stress induces cleavage of membrane-bound ATF6 by the same proteases that process SREBPs. Mol Cell. 2000;6:1355-64.

14. Tirasophon W, Welihinda AA, Kaufman RJ. A stress response pathway from the endoplasmic reticulum to the nucleus requires a novel bifunctional protein kinase/endoribonuclease (Ire1p) in mammalian cells. Genes Dev. 1998:12:1812-24.

15. Yoshida H, Haze K, Yanagi H, Yura T, Mori K. Identification of the cis-acting endoplasmic reticulum stress response element responsible for transcriptional induction of mammalian glucose-regulated proteins. 
Involvement of basic leucine zipper transcription factors. J Biol Chem. 1998; 273:33741-9.

16. Wang XZ, Harding HP, Zhang Y, Jolicoeur EM, Kuroda M, Ron D. Cloning of mammalian Ire1 reveals diversity in the ER stress responses. EMBO J. 1998; 17:5708-17.

17. Gardner BM, Walter P. Unfolded proteins are Ire1-activating ligands that directly induce the unfolded protein response. Science. 2011;333:1891-4.

18. Calfon M, Zeng H, Urano F, Till JH, Hubbard SR, Harding HP, et al. IRE1 couples endoplasmic reticulum load to secretory capacity by processing the XBP-1 mRNA. Nature. 2002;415:92-6.

19. Korennykh AV, Egea PF, Korostelev AA, Finer-Moore J, Zhang C, Shokat KM et al. The unfolded protein response signals through high-order assembly of Ire1. Nature. 2009;457:687-93.

20. Lu Y, Liang FX, Wang X. A synthetic biology approach identifies the mammalian UPR RNA ligase RtcB. Mol Cell. 2014;55:758-70.

21. Chen X, lliopoulos D, Zhang Q, Tang Q, Greenblatt MB, Hatziapostolou M, et al. XBP1 promotes triple-negative breast cancer by controlling the HIF1alpha pathway. Nature. 2014;508:103-7.

22. Lee AH, Scapa EF, Cohen DE, Glimcher LH. Regulation of hepatic lipogenesis by the transcription factor XBP1. Science. 2008;320:1492-6.

23. Ding L, Yan J, Zhu J, Zhong H, Lu Q, Wang Z, et al. Ligand-independent activation of estrogen receptor alpha by XBP-1. Nucleic Acids Res. 2003;31: 5266-74.

24. Martinon F, Chen X, Lee AH, Glimcher LH. TLR activation of the transcription factor XBP1 regulates innate immune responses in macrophages. Nat Immunol. 2010;11:411-8.

25. Hollien J, Weissman JS. Decay of endoplasmic reticulum-localized mRNAs during the unfolded protein response. Science. 2006;313:104-7.

26. Hatai T, Matsuzawa A, Inoshita S, Mochida Y, Kuroda T, Sakamaki K, et al. Execution of apoptosis signal-regulating kinase 1 (ASK1)-induced apoptosis by the mitochondria-dependent caspase activation. J Biol Chem. 2000;275 26576-81.

27. Nishitoh H, Saitoh M, Mochida Y, Takeda K, Nakano H, Rothe M, et al. ASK1 is essential for JNK/SAPK activation by TRAF2. Mol Cell. 1998;2:389-95.

28. Nishitoh H, Matsuzawa A, Tobiume K, Saegusa K, Takeda K, Inoue K, et al. ASK1 is essential for endoplasmic reticulum stress-induced neuronal cell death triggered by expanded polyglutamine repeats. Genes Dev. 2002;16: 1345-55.

29. Urano F, Wang X, Bertolotti A, Zhang Y, Chung P, Harding HP, et al. Coupling of stress in the ER to activation of JNK protein kinases by transmembrane protein kinase IRE1. Science. 2000;287:664-6.

30. Yan W, Frank CL, Korth MJ, Sopher BL, Novoa I, Ron D, et al. Control of PERK elF2alpha kinase activity by the endoplasmic reticulum stress-induced molecular chaperone P58IPK. Proc Natl Acad Sci U S A. 2002;99:15920-5.

31. Ladiges WC, Knoblaugh SE, Morton JF, Korth MJ, Sopher BL, Baskin CR, et al. Pancreatic beta-cell failure and diabetes in mice with a deletion mutation of the endoplasmic reticulum molecular chaperone gene P58IPK. Diabetes. 2005;54:1074-81.

32. van Huizen R, Martindale JL, Gorospe M, Holbrook NJ. P58IPK, a novel endoplasmic reticulum stress-inducible protein and potential negative regulator of elF2alpha signaling. J Biol Chem. 2003:278:15558-64.

33. Harding HP, Zhang Y, Ron D. Protein translation and folding are coupled by an endoplasmic-reticulum-resident kinase. Nature. 1999:397:271-4.

34. Pytel D, Majsterek I, Diehl JA. Tumor progression and the different faces of the PERK kinase. Oncogene. 2016:35:1207-15.

35. Bi M, Naczki C, Koritzinsky M, Fels D, Blais J, Hu N, et al. ER stress-regulated translation increases tolerance to extreme hypoxia and promotes tumor growth. EMBO J. 2005;24:3470-81.

36. Bobrovnikova-Marjon E, Grigoriadou C, Pytel D, Zhang F, Ye J, Koumenis C, et al. PERK promotes cancer cell proliferation and tumor growth by limiting oxidative DNA damage. Oncogene. 2010;29:3881-95.

37. Holcik M, Sonenberg N. Translational control in stress and apoptosis. Nat Rev Mol Cell Biol. 2005;6:318-27.

38. Cullinan SB, Zhang D, Hannink M, Arvisais E, Kaufman RJ, Diehl JA. Nrf2 is a direct PERK substrate and effector of PERK-dependent cell survival. Mol Cell Biol. 2003;23:7198-209.

39. Zhang W, Hietakangas V, Wee S, Lim SC, Gunaratne J, Cohen SM. ER stress potentiates insulin resistance through PERK-mediated FOXO phosphorylation. Genes Dev. 2013;27:441-9.

40. Malhi H, Kaufman RJ. Endoplasmic reticulum stress in liver disease. J Hepatol. 2011;54:795-809.
41. Chitnis NS, Pytel D, Bobrovnikova-Marjon E, Pant D, Zheng H, Maas NL, et al. miR-211 is a prosurvival microRNA that regulates chop expression in a PERK-dependent manner. Mol Cell. 2012;48:353-64.

42. Haze K, Yoshida H, Yanagi H, Yura T, Mori K. Mammalian transcription factor ATF6 is synthesized as a transmembrane protein and activated by proteolysis in response to endoplasmic reticulum stress. Mol Biol Cell. 1999;10:3787-99.

43. Szegezdi E, Logue SE, Gorman AM, Samali A. Mediators of endoplasmic reticulum stress-induced apoptosis. EMBO Rep. 2006;7:880-5.

44. Dalton LE, Clarke HJ, Knight J, Lawson MH, Wason J, Lomas DA, et al. The endoplasmic reticulum stress marker $\mathrm{CHOP}$ predicts survival in malignant mesothelioma. Br J Cancer. 2013;108:1340-7.

45. Kim KM, Yu TK, Chu HH, Park HS, Jang KY, Moon WS, et al. Expression of ER stress and autophagy-related molecules in human non-small cell lung cancer and premalignant lesions. Int J Cancer. 2012:131:E362-E70.

46. Davies MP, Barraclough DL, Stewart C, Joyce KA, Eccles RM, Barraclough R, et al. Expression and splicing of the unfolded protein response gene XBP-1 are significantly associated with clinical outcome of endocrine-treated breast cancer. Int J Cancer. 2008;123:85-8,

47. Wu H, Wei L, Fan F, Ji S, Zhang S, Geng J, et al. Integration of Hippo signalling and the unfolded protein response to restrain liver overgrowth and tumorigenesis. Nat Commun. 2015;6:6239.

48. Tang $\mathrm{CH}$, Ranatunga $\mathrm{S}$, Kriss $\mathrm{CL}$, Cubitt $\mathrm{CL}$, Tao J, Pinilla-lbarz JA, et al. Inhibition of ER stress-associated IRE-1/XBP-1 pathway reduces leukemic cell survival. J Clin Invest. 2014;124:2585-98.

49. Atkins C, Liu Q, Minthorn E, Zhang SY, Figueroa DJ, Moss K, et al. Characterization of a novel PERK kinase inhibitor with antitumor and antiangiogenic activity. Cancer Res. 2013;73:1993-2002.

50. Cross BC, Bond PJ, Sadowski PG, Jha BK, Zak J, Goodman JM, et al. The molecular basis for selective inhibition of unconventional mRNA splicing by an IRE1-binding small molecule. Proc Natl Acad Sci U S A. 2012;109:E869-78.

51. Ghosh R, Wang L, Wang ES, Perera BG, Igbaria A, Morita S, et al. Allosteric inhibition of the IRE1alpha RNase preserves cell viability and function during endoplasmic reticulum stress. Cell. 2014;158:534-48.

52. Axten JM, Romeril SP, Shu A, Ralph J, Medina JR, Feng Y, et al. Discovery of GSK2656157: an optimized PERK inhibitor selected for preclinical development. ACS Med Chem Lett. 2013;4:964-8.

53. Lee AH, Iwakoshi NN, Anderson KC, Glimcher LH. Proteasome inhibitors disrupt the unfolded protein response in myeloma cells. Proc Natl Acad Sci U S A. 2003:100:9946-51.

54. Shajahan AN, Riggins RB, Clarke R. The role of X-box binding protein-1 in tumorigenicity. Drug News Perspect. 2009;22:241-6.

55. Auf G, Jabouille A, Guerit S, Pineau R, Delugin M, Bouchecareilh M, et al. Inositol-requiring enzyme 1alpha is a key regulator of angiogenesis and invasion in malignant glioma. Proc Natl Acad Sci U S A. 2010;107:15553-8.

56. Chien W, Ding LW, Sun QY, Torres-Fernandez LA, Tan SZ, Xiao J, et al. Selective inhibition of unfolded protein response induces apoptosis in pancreatic cancer cells. Oncotarget. 2014;5:4881-94.

57. Maas NL, Diehl JA. Molecular pathways: the PERKs and pitfalls of targeting the unfolded protein response in cancer. Clin Cancer Res. 2015;21:675-9.

58. Nagelkerke A, Bussink J, Mujcic H, Wouters BG, Lehmann S, Sweep FC, et al. Hypoxia stimulates migration of breast cancer cells via the PERKATF4/ LAMP3-arm of the unfolded protein response. Breast Cancer Res. 2013;15:R2.

59. Mujcic H, Nagelkerke A, Rouschop KM, Chung S, Chaudary N, Span PN, et al. Hypoxic activation of the PERK/elF2alpha arm of the unfolded protein response promotes metastasis through induction of LAMP3. Clin Cancer Res. 2013;19:6126-37.

60. Morrow K, Hernandez CP, Raber P, Del VL, Wilk AM, Majumdar S, et al. Antileukemic mechanisms of pegylated arginase I in acute lymphoblastic T-cell leukemia. Leukemia. 2013;27:569-77.

61. Kusio-Kobialka M, Podszywalow-Bartnicka P, Peidis P, Glodkowska-Mrowka E, Wolanin K, Leszak G, et al. The PERK-elF2alpha phosphorylation arm is a pro-survival pathway of BCR-ABL signaling and confers resistance to imatinib treatment in chronic myeloid leukemia cells. Cell Cycle. 2012;11: 4069-78.

62. Donze $\mathrm{O}$, Jagus R, Koromilas AE, Hershey JW, Sonenberg N. Abrogation of translation initiation factor elF-2 phosphorylation causes malignant transformation of $\mathrm{NIH} 3 \mathrm{~T} 3$ cells. EMBO J. 1995;14:3828-34.

63. Sequeira SJ, Ranganathan AC, Adam AP, Iglesias BV, Farias EF, Aguirre-Ghiso JA. Inhibition of proliferation by PERK regulates mammary acinar morphogenesis and tumor formation. PLoS One. 2007;2:e615. 
64. Schardt JA, Weber D, Eyholzer M, Mueller BU, Pabst T. Activation of the unfolded protein response is associated with favorable prognosis in acute myeloid leukemia. Clin Cancer Res. 2009;15:3834-41.

65. Gabrilovich DI, Nagaraj S. Myeloid-derived suppressor cells as regulators of the immune system. Nat Rev Immunol. 2009;9:162-74.

66. Colegio OR, Chu NQ, Szabo AL, Chu T, Rhebergen AM, Jairam V, et al. Functional polarization of tumour-associated macrophages by tumourderived lactic acid. Nature. 2014;513:559-63.

67. Mahadevan NR, Rodvold J, Sepulveda H, Rossi S, Drew AF, Zanetti M. Transmission of endoplasmic reticulum stress and pro-inflammation from tumor cells to myeloid cells. Proc Natl Acad Sci USA. 2011;108:6561-6.

68. Zanetti M, Rodvold JJ, Mahadevan NR. The evolving paradigm of cellnonautonomous UPR-based regulation of immunity by cancer cells. Oncogene. 2016;35:269-78.

69. Yu Q, Zhao B, Gui J, Katlinski KV, Brice A, Gao Y, et al. Type I interferons mediate pancreatic toxicities of PERK inhibition. Proc Natl Acad Sci U S A. 2015;112:15420-5.

70. Deng J, Lu PD, Zhang Y, Scheuner D, Kaufman RJ, Sonenberg N, et al. Translational repression mediates activation of nuclear factor kappa B by phosphorylated translation initiation factor 2. Mol Cell Biol. 2004;24:10161-8.

71. Kitamura M. Biphasic, bidirectional regulation of NF-kappaB by endoplasmic reticulum stress. Antioxid Redox Signal. 2009;11:2353-64.

72. Pol J, Vacchelli E, Aranda F, Castoldi F, Eggermont A, Cremer I, et al. Trial Watch: Immunogenic cell death inducers for anticancer chemotherapy. Oncoimmunology. 2015;4:e1008866.

73. Lerner AG, Upton JP, Praveen PV, Ghosh R, Nakagawa Y, Igbaria A, et al. IRE1alpha induces thioredoxin-interacting protein to activate the NLRP3 inflammasome and promote programmed cell death under irremediable ER stress. Cell Metab. 2012;16:250-64.

74. Corzo CA, Cotter MJ, Cheng P, Cheng F, Kusmartsev S, Sotomayor E, et al. Mechanism regulating reactive oxygen species in tumor-induced myeloidderived suppressor cells. J Immunol. 2009;182:5693-701.

75. Jamora C, Dennert G, Lee AS. Inhibition of tumor progression by suppression of stress protein GRP78/BiP induction in fibrosarcoma B/C10ME. Proc Natl Acad Sci U S A. 1996:93:7690-4.

76. Pozzi C, Cuomo A, Spadoni I, Magni E, Silvola A, Conte A, et al. The EGFRspecific antibody cetuximab combined with chemotherapy triggers immunogenic cell death. Nat Med. 2016;22:624-31.

77. Thevenot PT, Sierra RA, Raber PL, Al-Khami AA, Trillo-Tinoco J, Zarreii P, et al. The stress-response sensor chop regulates the function and accumulation of myeloid-derived suppressor cells in tumors. Immunity. 2014;41:389-401.

78. Reimold AM, Iwakoshi NN, Manis J, Vallabhajosyula P, Szomolanyi-Tsuda E, Gravallese EM, et al. Plasma cell differentiation requires the transcription factor XBP-1. Nature. 2001;412:300-7.

79. Bettigole SE, Lis R, Adoro S, Lee AH, Spencer LA, Weller PF, et al. The transcription factor XBP1 is selectively required for eosinophil differentiation. Nat Immunol. 2015;16:829-37.

80. Iwakoshi NN, Pypaert M, Glimcher LH. The transcription factor XBP-1 is essential for the development and survival of dendritic cells. J Exp Med. 2007;204:2267-75.

81. Hu R, Chen ZF, Yan J, Li QF, Huang Y, Xu H, et al. Endoplasmic Reticulum Stress of Neutrophils Is Required for Ischemia/Reperfusion-Induced Acute Lung Injury. J Immunol. 2015;195:4802-9.

82. Herber DL, Cao W, Nefedova Y, Novitskiy SV, Nagaraj S, Tyurin VA, et al. Lipid accumulation and dendritic cell dysfunction in cancer. Nat Med. 2010;16:880-6.

83. Ramakrishnan $\mathrm{R}$, Tyurin VA, Veglia F, Condamine T, Amoscato A, Mohammadyani $D$, et al. Oxidized lipids block antigen cross-presentation by dendritic cells in cancer. J Immunol. 2014;192:2920-31.

84. Hossain F, Al-Khami AA, Wyczechowska D, Hernandez C, Zheng L, Reiss K, et al. Inhibition of fatty acid oxidation modulates immunosuppressive functions of myeloid-derived suppressor cells and enhances cancer therapies. Cancer Immunol Res. 2015;3:1236-47.

85. Condamine T, Dominguez GA, Youn JI VKA, Mony S, Alicea-Torres K, et al. Lectin-type oxidized LDL receptor-1 distinguishes population of human polymorphonuclear myeloid-derived suppressor cells in cancer patients. Sci Immunol. 2016;1:aaf8943.

86. Yan D, Wang HW, Bowman RL, Joyce JA. STAT3 and STAT6 signaling pathways synergize to promote cathepsin secretion from macrophages via IRE1alpha activation. Cell Rep. 2016:16:2914-27.
87. Goodall JC, Wu C, Zhang Y, McNeill L, Ellis L, Saudek V, et al. Endoplasmic reticulum stress-induced transcription factor, $\mathrm{CHOP}$, is crucial for dendritic cell IL-23 expression. Proc Natl Acad Sci USA. 2010;107:17698-703.

88. Chen L, Jarujaron S, Wu X, Sun L, Zha W, Liang G, et al. HIV protease inhibitor lopinavir-induced TNF-alpha and IL-6 expression is coupled to the unfolded protein response and ERK signaling pathways in macrophages. Biochem Pharmacol. 2009;78:70-7.

89. Oh J, Riek AE, Weng S, Petty M, Kim D, Colonna M, et al. Endoplasmic reticulum stress controls M2 macrophage differentiation and foam cell formation. J Biol Chem. 2012;287:11629-41.

90. Cubillos-Ruiz JR, Glimcher LH. Targeting abnormal ER stress responses in tumors: a new approach to cancer immunotherapy. Oncoimmunology. 2016;5:e1098802.

91. Axten JM, Medina JR, Feng Y, Shu A, Romeril SP, Grant SW, et al. Discovery of 7-methyl-5-(1-\{[3-(trifluoromethyl)phenyl]acetyl\}-2,3-dihydro-1H-indol-5yl)-7H-p yrrolo[2,3-d]pyrimidin-4-amine (GSK2606414), a potent and selective first-in-class inhibitor of protein kinase R (PKR)-like endoplasmic reticulum kinase (PERK). J Med Chem. 2012;55:7193-207.

92. Gajewski TF, Schreiber H, Fu YX. Innate and adaptive immune cells in the tumor microenvironment. Nat Immunol. 2013:14:1014-22.

\section{Submit your next manuscript to BioMed Central and we will help you at every step:}

- We accept pre-submission inquiries

- Our selector tool helps you to find the most relevant journal

- We provide round the clock customer support

- Convenient online submission

- Thorough peer review

- Inclusion in PubMed and all major indexing services

- Maximum visibility for your research

Submit your manuscript at www.biomedcentral.com/submit
) Biomed Central 